\title{
Vibration response imaging versus perfusion scan in lung cancer surgery evaluation
}

\author{
Nuria Marina, MD, ${ }^{\text {a }}$ Gema Rodriguez-Trigo, MD, ${ }^{\text {,c }}$ Unai Jimenez, MD, ${ }^{\mathrm{d}}$ Beatriz Morales, MD, \\ Elena López de Santa María, ${ }^{\mathrm{a}}$ José Ignacio Pijoan, MD, ${ }^{\mathrm{e}}$ and Juan B. Gáldiz, MD, PhD ${ }^{\mathrm{a}, \mathrm{f}}$
}

Objective: Ventilation/perfusion scan is a standard procedure in high-risk surgical patients to predict pulmonary function after surgery. Vibration response imaging is a technique that could be used in these patients. The objective of our study was to compare this imaging technique with the usual scanning technique for predicting postoperative forced expiratory volume.

\begin{abstract}
Methods: We assessed 48 patients with lung cancer who were candidates for lung resection. Forced spirometry, vibration response imaging, and ventilation/perfusion scan were performed in patients before surgery, and spirometry was performed after intervention.

Results: We included 48 patients (43 men; mean age, 64 years) undergoing lung cancer surgery (32 lobectomies/ 16 pneumonectomies). On comparison of both techniques, for pneumonectomy, we found a concordance of 0.84 (95\% confidence interval, 0.76-0.92) and Bland-Altman limits of agreement of -0.33 to +0.45 , with an average difference of 0.064 . By comparing postoperative spirometry with vibration response imaging, we found a concordance of 0.66 (95\% confidence interval, 0.38-0.93) and Bland-Altman limits of agreement of -0.60 to +0.33 , with an average difference of -0.13 .
\end{abstract}

Conclusions: The 2 techniques presented good concordance values. Vibration response imaging shows non-negligible confidence intervals. Vibration response imaging may be useful in preoperative algorithms in patients before lung cancer surgery. (J Thorac Cardiovasc Surg 2014;147:816-21)

Lung surgery remains the best therapeutic option for patients diagnosed with lung cancer and may lead to full recovery from the disease. However, deciding whether a patient should undergo lung resection requires a thorough assessment of lung function, frequently through the combination of different techniques that can predict surgical risk and postoperative lung function.

Available guidelines ${ }^{1-3}$ for the evaluation of patients with lung cancer for radical surgery recommend different techniques, such as spirometry, diffusing capacity of the lung for carbon monoxide (DLCO), and exercise tests. When the parameters measured are below an agreed threshold, ventilation/perfusion scintigraphy (V/Q) should be performed.

V/Q techniques currently tend to be considered the reference gold standard to obtain predicted postoperative

From the Department of Pulmonology, ${ }^{\text {a }}$ Cruces University Hospital, Basque Country, Spain; Department of Pulmonology, ${ }^{\mathrm{b}}$ San Carlos Hospital, Madrid, Spain; Complutense University, ${ }^{\mathrm{c}}$ Madrid, Spain; Department of Thoracic Surgery, ${ }^{\mathrm{d}}$ Cruces University Hospital, Basque Country, Spain; Epidemiology Department, ${ }^{\text {e }}$ Cruces University Hospital, Basque Country, Spain; and CibeRes and Basque Country University, ${ }^{\mathrm{f}}$ Basque Country, Spain.

Disclosures: Authors have nothing to disclose with regard to commercial support.

Received for publication Feb 1, 2013; revisions received Aug 5, 2013; accepted for publication Aug 22, 2013; available ahead of print Oct 29, 2013.

Address for reprints: Nuria Marina, MD, Pneumology Department, Cruces University Hospital, $2^{\mathrm{a}} \mathrm{D}$. Plaza de Cruces, s/n, 48903 Baracaldo, Vizcaya, Spain (E-mail: nuria.marinamalanda@osakidetza.net).

$0022-5223 / \$ 36.00$

Copyright (c) 2014 by The American Association for Thoracic Surgery

http://dx.doi.org/10.1016/j.jtcvs.2013.08.066 forced expiratory volume in 1 second (ppoFEV1). This value is estimated on the basis of the forced expiratory volume in 1 second (FEV1) before surgery, the extent of the planned resection, and the contribution of each lung segment in terms of ventilation or perfusion. This approach is expensive and complex, and V/Q scans have to be carried out in the Nuclear Medicine Department, requiring the administration of radioisotopes and radiation.

Vibration response imaging (VRI) is a new, noninvasive, simple to use technique that can provide an accurate estimate of ppoFEV $1 .{ }^{4}$ In a previous study by Jimenez and colleagues, ${ }^{5}$ VRI-based measures have shown high accuracy in the prediction of ppoFEV1.

The hypothesis of this study is that VRI might have as good a predictive capacity of the ppoFEV1 as V/Q scan, and therefore our main objective was to compare the 2 techniques in patients with lung cancer who are candidates for lung resection.

\section{MATERIALS AND METHODS}

We conducted a prospective study that included all patients with lung cancer evaluated for lung resection before surgery, regardless of the degree of severity. All patients were first assessed by a multidisciplinary committee for lung cancer.

The study was performed at Cruces University Hospital (Bizkaia) and San Carlos Hospital (Madrid), Spain, during 9 months in 2009. During the course of the study, 63 patients were evaluated, of whom 48 underwent operation and were recruited (5 women and 43 men) (Table 1). Fifteen patients did not undergo surgery for various reasons (mostly no surgical stage). This study was approved by the ethics committees of 


\section{Abbreviations and Acronyms \\ CI $=$ confidence interval \\ $\mathrm{CT}=$ computed tomography \\ DLCO = diffusing capacity of the lung for carbon monoxide \\ FEV1 = forced expiratory volume in 1 second \\ ppoFEV1 $=$ predicted postoperative forced expiratory volume in 1 second \\ $\mathrm{V} / \mathrm{Q}=$ ventilation/perfusion scintigraphy \\ VRI $=$ vibration response imaging}

both participating hospitals, and written informed consent was obtained from all participants.

\section{Assessment of Patients}

Patients were assessed in the Respiratory Function Units of each hospital. The assessment algorithm is depicted in Figure 1.

All 48 patients subsequently underwent surgery (lobectomy in 32 patients and pneumonectomy in 16 patients). Preoperative tests included spirometry, V/Q scan, and VRI to estimate the ppoFEV1. Spirometry was performed 4 to 6 weeks after surgery to assess the postoperative FEV1. This period of time was considered to be sufficiently prolonged to accurately assess the postoperative lung function of patients before the use of potential coadjuvant therapy.

\section{Spirometry}

Spirometry was performed using a spirometer Master Scope Jaeger (Wulzurg, Germany) in accordance with the American Thoracic Society/European Respiratory Society ${ }^{6}$ procedure standards. All tests were performed by the same technician in each hospital.

\section{Pulmonary Ventilation/Perfusion Scintigraphy}

V/Q scans were performed in all patients using a gamma camera (InfiniaTM; General Electric Healthcare, Little Chalfont, Buckinghamshire, UK) and following standard procedures: Patients inhaled $30 \mathrm{mCi}$ 99mTc-DTPA for ventilation assessment and were administered intravenous 99Tc-MAA for the perfusion images. Obtained images show the lung divided into 3 similar segments, with each area contributing a different percentage in terms of perfusion and ventilation.

To calculate ppoFEV1, we used the equations given in the study by Wernly and colleagues ${ }^{7}$ :

Predicted postoperative FEV1 = preoperative FEV1 $\times(1 \%$ Q of lung to be resected) for perfusion scintigraphy;

Predicted postoperative FEV1 = preoperative FEV1 $\times(1 \%$ V of lung to be resected) for ventilation scintigraphy; and

Predicted postoperative FEV1 = preoperative FEV $1 \times \%$ matched V/ $\mathrm{Q}$ for combined V/Q scintigraphy.

\section{Vibration Response Imaging}

A pre-surgery VRI protocol previously described by our group ${ }^{5}$ was applied to all patients. Briefly, the VRI system (VRIxpTM; Deep Breeze Ltd, Or-Akiva, Israel) evaluates pulmonary function by recording the sounds of consecutive regular respiratory cycles ( 3 to 5 for a period of 12 seconds), measuring the energy produced by the vibration of air as it passes through the airways. These vibrations are picked up by 2 arrays of sensors that are placed on the patient's back and via low vacuum cups. Dedicated software creates dynamic images of each lung and the signals are converted into percentages, reflecting the contribution of each segment to the vibrations during breathing. Each lung is divided into 3 segments, as in V/Q scan, so that this new technique allows the segmental counting as V/Q scan. The technique must be carried out in total silence, with no episodes of coughing or talking during the recordings, because these would interfere with the signal measurement process.

Once the respiratory cycles were recorded, the data were evaluated using the O-Plan software (Deep Breeze Ltd). The technician selected the most suitable and similar cycles to achieve the best possible data, with a standard deviation no more than $5 \%$ between them. The O-Plan software, using the selected cycles and FEV1 before surgery, calculates ppoFEV1.

\section{Statistical Analysis}

Our aim was to assess the relationship and degree of agreement among predictions of ppoFEV1 obtained using these 2 methods (VRI and V/Q scan) and compare the degree of agreement of these 2 estimates with the actual postoperative FEV1. To do so, we used 2 complementary tools, Lin's ${ }^{8}$ concordance correlation coefficient, a numeric index ranging from 0 to 1 , and Bland-Altman plots with average difference between measures and $95 \%$ limits of agreement. ${ }^{9}$ For concordance coefficient, $95 \%$ confidence intervals (CIs) were calculated for concordance coefficient. We followed the agreement strength classification proposed by McBride. ${ }^{10}$

Statistical analysis was carried out using the Statistical Package for the Social Sciences (version 11; 5. 1, SPSS, Inc, Chicago, Ill) and Stata 11 for Windows (2009 Stata Statistical Software: Release 11; StataCorp LP, College Station, Tex).

\section{RESULTS}

Forty-eight patients undergoing lung resection were included in our study, of whom 32 had lobectomies and 16 had pneumonectomies.

1. Predicted postoperative FEV1 by both techniques (Figure 2): For a theoretic pneumonectomy in all patients (48 patients), we found a concordance coefficient of 0.84 (95\% CI, 0.76-0.92) in absolute values and 0.73 (95\% CI, 0.59-0.86) in percentage. In case of lobectomy as the planned operative approach (32 patients), the concordance was 0.88 (95\% CI, 0.81-0.95) in absolute values and 0.75 (95\% CI, 0.62-0.89) in percentage.

2. Concordance between postoperative FEV1 predicted with both techniques and observed postoperative FEV1:

a. ppoFEV1 by V/Q scan versus postoperative FEV1 (Figure 3): For actual pneumonectomy (16 patients), we obtained a concordance coefficient of $0.80(95 \%$ CI, 0.61-0.98) in absolute values and 0.67 (95\% CI, 0.40-0.93) in percentage. In lobectomy cases (32 patients), a concordance of 0.81 (95\% CI, $0.70-0.93$ ) was found for absolute values and 0.70 (95\% CI, 0.52-0.87) in percentage.

b. ppoFEV1 by VRI versus postoperative FEV1 (Figure 4): In pneumonectomy cases (16 patients), we obtained a concordance of 0.66 (95\% CI, 0.38-0.93) in absolute values and 0.52 (95\% CI, 0.21-0.83) in percentage. In lobectomy cases (32 patients), concordance was 0.81 (95\% CI, 0.68-0.93) for absolute values and 0.60 (95\% CI, 0.38-0.82) in percentage. 
TABLE 1. Characteristics of patients ( \pm numbers represent standard deviations)

\begin{tabular}{|c|c|c|}
\hline \multicolumn{2}{|l|}{ No. of patients } & 48 \\
\hline \multicolumn{2}{|c|}{ Mean age, y (minimum-maximum) } & $64.02(45-83)$ \\
\hline \multicolumn{2}{|l|}{ Women:men } & $5: 43$ \\
\hline \multicolumn{2}{|c|}{ Preoperative FEV1 } & $2.01 \pm 0.57(68.5 \% \pm 15.03 \%)$ \\
\hline \multicolumn{2}{|c|}{ Type of surgery (n) } & Pneumonectomy (16) \\
\hline \multirow[t]{2}{*}{$\begin{array}{l}\text { Postoperative } \\
\text { FEV1 }\end{array}$} & $\begin{array}{l}\text { Pneumonectomy } \\
\text { (16 patients) }\end{array}$ & $1.40 \pm 0.301(45.33 \% \pm 7.09 \%)$ \\
\hline & $\begin{array}{l}\text { Lobectomy } \\
\text { (32 patients) }\end{array}$ & $1.68 \pm 0.511(56.6 \% \pm 12.81 \%)$ \\
\hline
\end{tabular}

FEV1, Forced expiratory volume in 1 second.

\section{DISCUSSION}

The aim of this study was to assess agreement and compare predictions of postoperative FEV1 produced by a well-established method, V/Q scan, and a novel technique, VRI imaging.

We have prospectively examined all candidates for lung cancer surgery, regardless of functional severity, and assessed the concordance between these 2 techniques when estimating the postoperative FEV1. In addition, we estimated concordance indexes with the FEV1 after surgery.

Our results indicate that there is substantial degree of concordance between predictions from VRI and V/Q scan in candidates for surgery, regardless of the type of treatment proposed, pneumonectomy or lobectomy. Furthermore, in the group of patients who actually underwent surgery, the concordance values obtained were acceptable, comparing

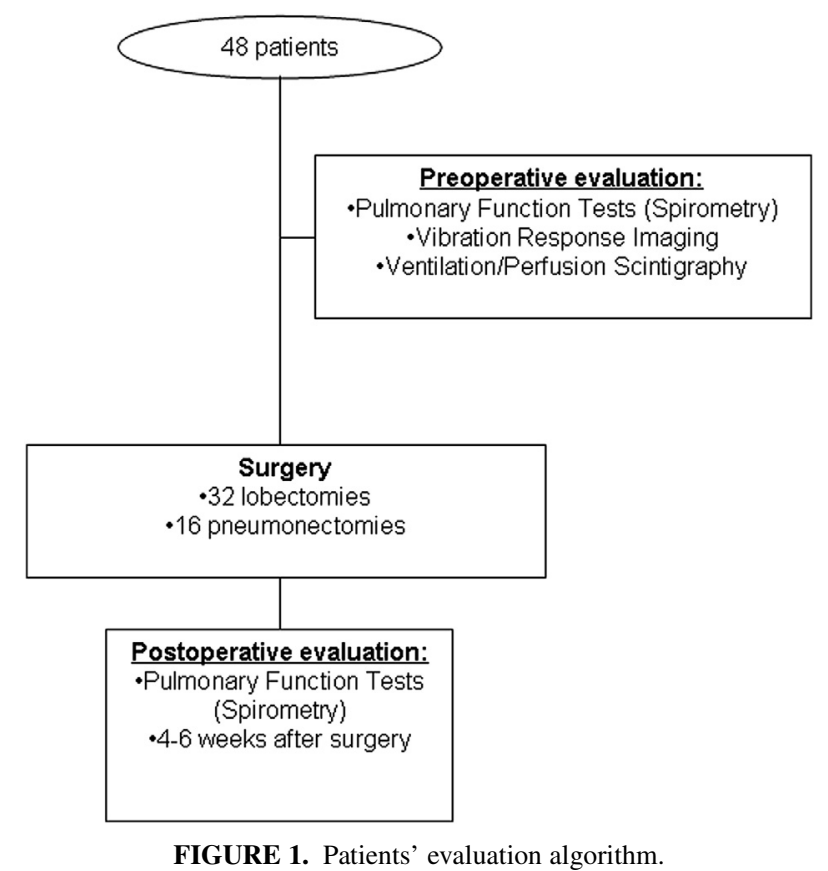

the predictions obtained with both techniques and the postoperative FEV1. Inaccuracy in predicting postlobectomy FEV1 is usually greater, because anatomic limits of resection are difficult to approach by 2-dimensional images provided by V-Q scan and VRI devices. This fact can be deduced from our results and is easily recognized in Figures 2 and 3. Another suggested approach is prediction based on the count of resected functional segments. This more anatomic method relies on the supposition that all segments contribute similarly to lung function. This assumption is less realistic in patients with chronic obstructive pulmonary disorder, who comprise the majority of patients with pulmonary function near the limits of resectability, because functional distribution, both ventilation and perfusion, may be more heterogeneous. An intermediate solution is to give segment pretended to resect a coefficient dependent on gammagraphic distribution.

The recently published preoperative evaluation guidelines concerning pulmonary resection for lung cancer ${ }^{1-3}$ recommend that spirometry and diffusion tests should be performed in all candidates and complemented with exercise tests when the FEV1 or DLCO is less than $80 \%$ of predicted. If patients do not reach a level of oxygen consumption of $20 \mathrm{~mL} / \mathrm{kg} / \mathrm{min}$ or $75 \%$ of predicted during the cardiorespiratory exercise test, the study should be completed using V/Q to calculate ppoFEV1. In the current study, FEV1 was the parameter aimed at and not DLCO, which is included in the new guidelines.

V/Q scan was first described in 1974 as a diagnostic test to assess the FEV1 after surgery. ${ }^{11}$ Published correlations between postoperative FEV1 and predictions using ventilation or perfusion scan vary widely among different studies. Win and colleagues ${ }^{12}$ studied a series of 32 patients (21 men and 11 women) during a 24-month period, comparing ventilation and perfusion scan results with the postoperative FEV1, and reported correlation values of 0.70 and 0.58 for ventilation and perfusion, respectively. Sangalli and colleagues ${ }^{13}$ compared the predictions of perfusion scintigraphy with postsurgical spirometry in 40 patients during a period of 27 months. The correlations for pneumonectomy and lobectomy were 0.89 and 0.78 , respectively.

Several studies have found better correlations using ventilation than perfusion scan. Ladurie and RansonBitker $^{14}$ reported correlations of 0.74 and 0.70 for ventilation and perfusion, respectively, based on a sample of 159 patients (137 men and 22 women) who underwent pneumonectomy. Perfusion scintigraphy is the most widely used method to predict ppoFEV1, although there is not a clear consensus in the scientific literature. In our study, we performed both ventilation and perfusion scan and used the values obtained by perfusion as reference, with 


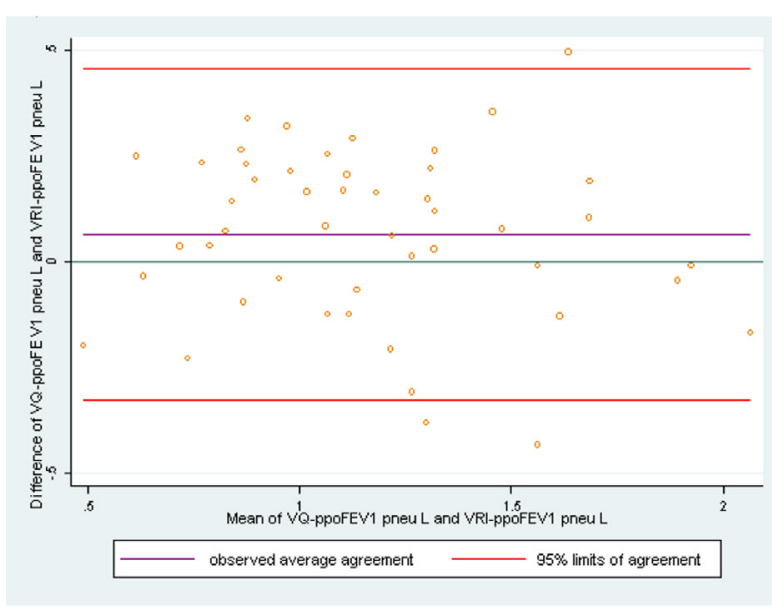

A

\section{A} (VRI-ppoFEV1 pneu L) (Bland-Altman average difference, 0.06 ; 95\% CI, -0.33 to 0.45 ) for patients undergoing pneumonectomy and (B) between VQ (VQ-ppoFEV1 lobect L) and VRI (VRI-ppoFEV1 lobect L) (Bland-Altman average difference, 0.18; 95\% CI, 0.20-0.57) for patients undergoing lobectomy. ppoFEV1, Predicted postoperative forced expiratory volume in 1 second; $V Q$, ventilation/perfusion scintigraphy; VRI, vibration response imaging.

correlation values similar to those reported by other authors. $^{12-14}$

Given these limitations of V/Q scan, other techniques for predicting postoperative FEV1 have been evaluated. Among these, magnetic resonance imaging has been used to provide dynamic perfusion imaging with good results. Ohno and colleagues ${ }^{15}$ assessed 60 patients using spirometry before and after surgery, and magnetic resonance imaging and perfusion scan to estimate the ppoFEV1 before surgery. They found a better correlation with postoperative FEV1 (0.93) than perfusion scan (0.89). Other imaging techniques, such as quantitative computed tomography (CT), also have been used to

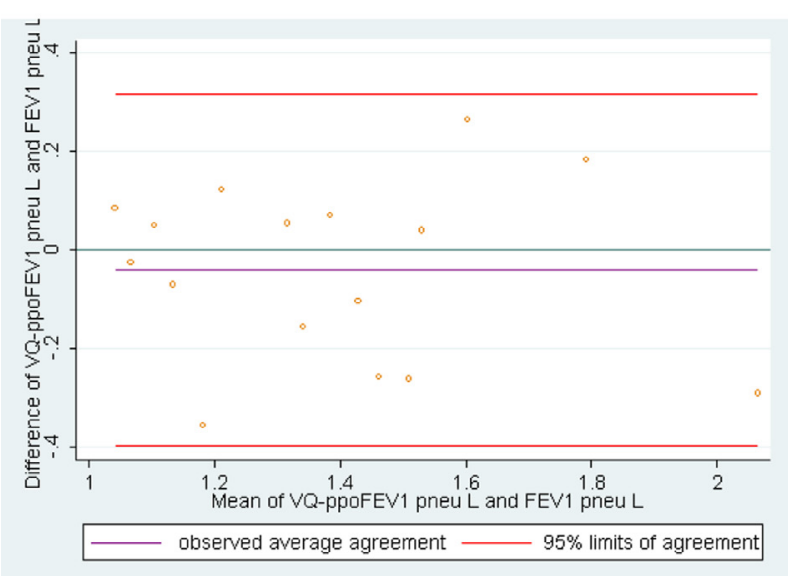

\section{A}

FIGURE 3. Concordance and Bland-Altman plots of postoperative predictions in absolute values (liters) (A) between VQ (VQ-ppoFEV1 pneu L) and FEV1 postpneumonectomy (FEV1 pneu L) (Bland-Altman average difference, $-0.04 ; 95 \%$ CI, -0.40 to 0.32 ) for patients undergoing pneumonectomy and (B) between VQ (VQ-ppoFEV1 lobect L) and FEV1 postlobectomy (FEV1 lobect L) (Bland-Altman average difference, 0.16; 95\% CI, 0.41-0.72) for patients undergoing lobectomy. ppoFEV1, Predicted postoperative forced expiratory volume in 1 second; $V Q$, ventilation/perfusion scintigraphy; FEV1, forced expiratory volume in 1 second. quantify ppoFEV1. The study by Bolliger and colleagues ${ }^{16}$ evaluated 5 different techniques in 44 patients: perfusion scan, quantitative CT scan, and 3 anatomy-based calculations. They found correlations of 0.92 for the perfusion scan, 0.91 for the CT scan, and 0.88 for the 3 anatomic equations. $\mathrm{Wu}$ and colleagues ${ }^{17}$ compared the correlations between perfusion scintigraphy and CT predictions in 44 patients and found values of 0.88 and 0.86 , respectively, for patients who underwent pneumonectomy and 0.9 and 0.80 , respectively, for patients who underwent lobectomy.

More complex techniques have been investigated, such as single-photon emission CT. Mineo and colleagues ${ }^{18}$ recently reported correlations of 0.83 to 0.85 . These authors

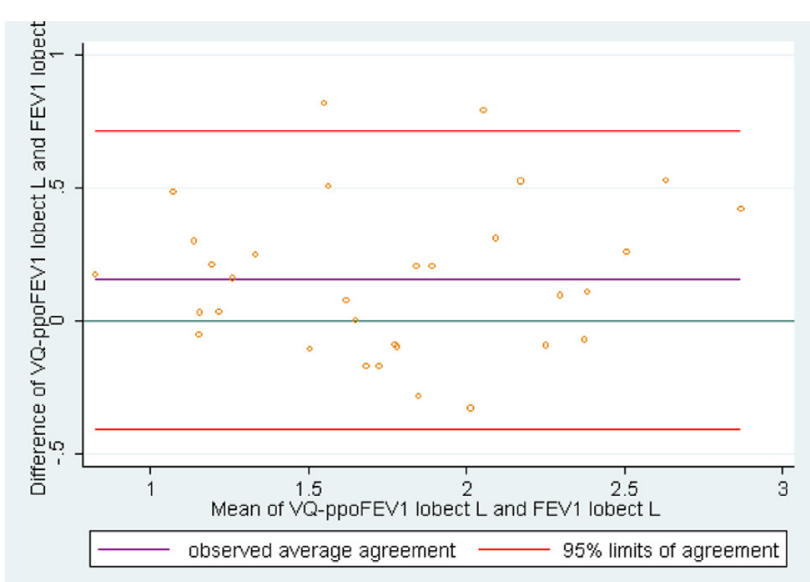

$\mathbf{B}$ 


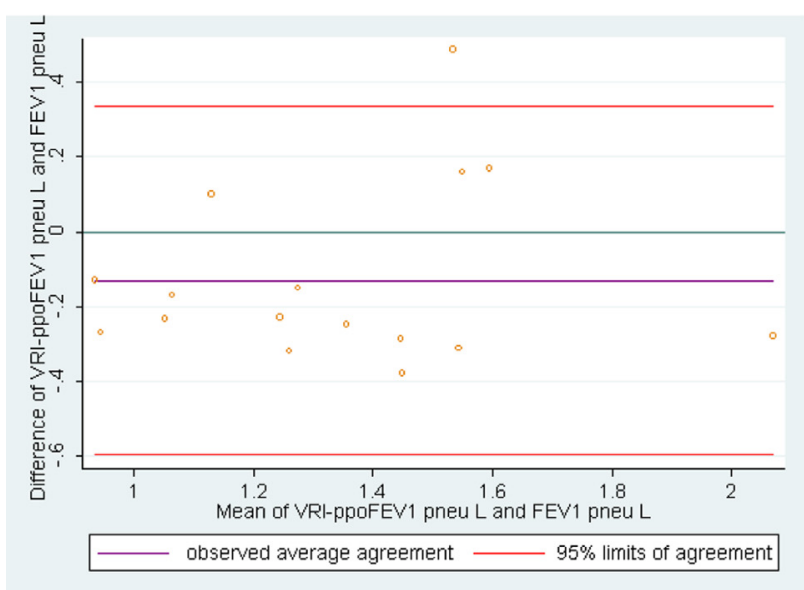

A

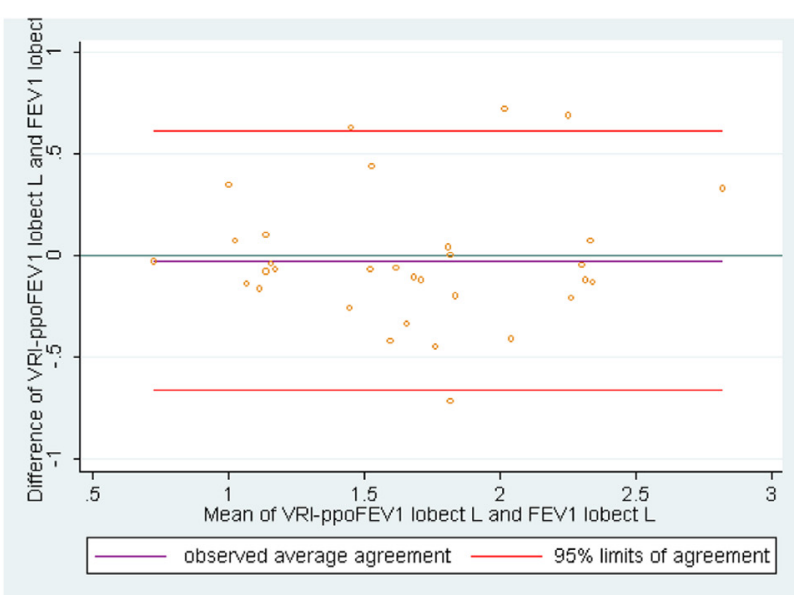

B

FIGURE 4. Concordance and Bland-Altman plots of postoperative predictions in absolute values (liters) (A) between VRI (VRI-ppoFEV1 pneu L) and FEV1 postpneumonectomy (FEV1 pneu L) (Bland-Altman average difference, -0.13 ; 95\% CI, $-0.60-0.33$ ) for patients undergoing pneumonectomy and (B) between VRI (VRI-ppoFEV1 lobect L) and FEV1 postlobectomy (FEV1 lobect L) (Bland-Altman average difference, -0.02 ; 95\% CI, 0.66/0.61) for patients undergoing lobectomy. ppoFEV1, Predicted postoperative forced expiratory volume in 1 second; VRI, vibration response imaging; FEV1, forced expiratory volume in 1 second.

studied the pulmonary function of 39 patients and compared prediction using single-photon emission CT before surgery with measurements after surgery.

VRI is a simple, noninvasive test that does not involve ionizing radiation or any special preparation by the patients. It has been used for various different respiratory conditions (eg, mechanical ventilation, ${ }^{19}$ pneumonia, pleural effusion, ${ }^{20}$ lung transplantation, ${ }^{21}$ and asthma $^{22}$ ). Some studies have assessed the images produced by healthy individuals ${ }^{4,23}$ to compare them with those observed in various different respiratory diseases, both in outpatients and in individuals in the intensive care or emergency units, ${ }^{23}$ where patients are lying down.

Previous studies have demonstrated that VRI may be useful in the preoperative evaluation in patients with lung cancer. Jimenez and colleagues ${ }^{5}$ studied 58 patients who underwent lung resection surgery (12 pneumonectomies and 46 lobectomies) evaluating the spirometric postoperative FEV1 with ppoFEV1 obtained by VRI. They found a correlation of 0.86 (absolute values) and 0.87 (percentages), with a concordance of 0.85 and 0.86 , respectively. Comce and colleagues ${ }^{24}$ recently found similar results for this technique.

We have not found any study that provides concordance values between actual postoperative FEV1 and ppoFEV1 values obtained by different techniques. Most often, studies report correlation coefficients that may miss important disagreement in studies to compare methods. Although no method comparison index is free of limitations, it has been advised to combine several approaches. ${ }^{25}$ To follow this suggestion, we have combined a commonly used graphic tool to show relationships between 2 quantitative measurements (Bland-Altman limits of agreement plot) and a quantitative index (Lin's ${ }^{8}$ concordance correlation coefficient), which is related to Pearson correlation coefficient but with the added value that it can also account for the presence of systematic differences between methods.

To make our results more comparable with previous research on this issue, we have also calculated standard correlation coefficients between the 2 techniques obtaining a value of 0.86 and 0.93 between VRI and V/Q scan in absolute values for possible pneumonectomy and lobectomy, respectively. By comparing V/Q scan with the postoperative FEV1, the correlation was 0.81 for pneumonectomy and 0.85 for lobectomy in absolute values. By using VRI and postoperative FEV1, the values obtained were 0.72 and 0.81 for pneumonectomy and lobectomy, respectively. These values are similar to those found in other studies using this approach.

Our VRI data overall show moderate to substantial agreement with postoperative FEV1 and V/Q scan measurements. However, the amplitude of the CI for concordance index together with apparent differences between both measures in individual cases shown by Bland-Altman plots prompt us to be cautious regarding straight exchangeability of both predictive techniques. This is illustrated by the width of the limits of agreement. This issue calls for further insight into potential sources of variability (noise) in measurements and patient selection criteria. Our study provides new evidence to support that VRI could be included in preoperative evaluation algorithms for patients with lung cancer.

The VRI technique has several advantages. Results are obtained quickly; only 4 to 6 minutes are required to perform the test, and the O-Plan software analysis takes 
approximately 5 minutes. VRI can be performed in different settings because the equipment is easy to transport and handle. In contrast, the V/Q scan must be carried out in departments/units of nuclear medicine because it is based on radiation and the administration of radioisotopes, both inhaled and intravenously. In addition, it is expensive, uncomfortable, and complex to perform for the patient.

Technical limitations associated with the use of VRI include very thin patients and those having a hairy back, which makes it difficult to place the suction cups. However, these problems can be addressed, because patients' backs can be shaved and a pillow or cushion can be given for patients to "hug" so that their shoulder blades are moved forward, thus solving the problem. In our study, no patient was excluded because of these potential problems. Other potential limitations include noise taking place in patients who have pacemakers or mechanical heart valves, which can interfere with the recordings.

\section{CONCLUSIONS}

Our study provides further evidence that VRI measurements can yield useful predictions of postoperative lung function. In addition, these predictions are obtained in a rapid, comfortable, and inexpensive way. Further research into specific aspects, such as modifiable sources of variability and proper patient selection, is warranted to determine whether this technique is a real alternative to VQ scan, the current reference test.

\section{References}

1. Brunelli A, Charloux A, Bolliger C, Rocco G, Sculler JP, Varela G, et al. ERS/ESTS clinical guidelines on fitness for radical therapy in lung cancer patients (surgery and chemo-radiotherapy). Eur Respir J. 2009;34:17-41.

2. British Thoracic Society, Society of Cardiothoracic Surgeons of Great Britain and Ireland Working Party. Guidelines on the selection of patients with lung cancer for surgery. Thorax. 2001;56:89-108.

3. The American Thoracic Society and The European Respiratory Society. Pretreatment evaluation of non-small-cell lung cancer. Am J Respir Crit Care Med. 1997;156:320-2.

4. Yigla M, Gat M, Meyer JJ, Friedman PJ, Maher TM, Madison JM. Vibration response imaging technology in healthy subjects. AJR Am J Roentgenol. 2008; 191:845-52.

5. Jimenez U, Marina N, Lopez de Santamaría E, Pac JJ, Galdiz JB. Evaluation of the utility of vibration response imaging device and operation planning software in the assessment of patients before lung resection surgery. Eur J Cardiothorac Surg. 2010;37:1185-90.
6. Miller MR, Hankinson J, Brusasco V, Burgos F, Casaburi R, Coates A, et al. ATS/ ERS Task Force. Standardisation of spirometry. Eur Respir J. 2005;26:319-38.

7. Wernly JA, Demeester TR, Kirchner PT, Myerowitz PD, Oxford DE, Golomb HM. Clinical value of quantitative ventilation-perfusion lung scan in the surgical management of bronchogenic carcinoma. J Thorac Cardiovasc Surg. 1980;80:535-43.

8. Lin L. A concordance correlation coefficient to evaluate reproducibility. Biometrics. 1989;45:255-68.

9. Bland JM, Altman DG. Statistical methods for assessing agreement between two methods of clinical measurement. Lancet. 1986;1:307-10.

10. McBride GB. A proposal for strength-of-agreement criteria for Lin's concordance correlation coefficient. Hamilton, New Zealand: National Institute of Water \& Atmospheric Research Ltd; 2005. Available at: http://www.medcalc. org/download/pdf/McBride2005.pdf. Accessed May 2005.

11. Olsen GN, Block AJ, Tobias JA. Prediction of postpneumonectomy pulmonary function using quantitative macroaggregate lung scanning. Chest. 1974;66:13-6.

12. Win T, Tasker AD, Groves AM, White C, Ritchie AJ, Wells FC, et al. Ventilationperfusion scintigraphy to predict postoperative pulmonary function in lung cancer patients undergoing pneumonectomy. AJR Am J Roentgenol. 2006;187:1260-5.

13. Sangalli M, Spiliopoulos A, Mégevand RR. Predictability of FEVl after pulmonary resection for bronchogenic carcinoma. Eur J Cardiothorac Surg. 1992;6:242-5.

14. Ladurie ML, Ranson-Bitker B. Uncertainties in the expected value for forced expiratory volume in one second after surgery. Chest. 1986;90:222-8.

15. Ohno Y, Hatabu H, Higashino T, Takenaka D, Watanabe H, Nishimura Y, et al. Dynamic perfusion MRI versus perfusion scintigraphy: prediction of postoperative lung function in patients with lung cancer. AJR Am J Roentgenol. 2004;182:73-8.

16. Bolliger CT, Gückel C, Engel H, Stöhr S, Wyser CP, Schoetzau A, et al. Prediction of functional reserves after lung resection: comparison between quantitative computed tomography, scintigraphy and anatomy. Respiration. 2002;69:482-9.

17. Wu MT, Pan HB, Chiang AA, Hsu HK, Chang HC, Peng NJ, et al. Prediction of postoperative lung function in patients with lung cancer: comparison of quantitative CT with perfusion scintigraphy. AJR Am J Roentgenol. 2002;178:667-72.

18. Mineo TC, Schillazi O, Pompeo E, Mineo D, Simonetti G. Usefulness of lung perfusion scintigraphy before lung cancer resection in patients with ventilatory obstruction. Ann Thorac Surg. 2006;82:1828-34.

19. Jean S, Cinel I, Gratz I, Tay C, Lotano V, Deal E, et al. Image-based monitoring of one lung ventilation with vibration response imaging. Eur J Anaesthesiol. 2008; 25:995-1001.

20. Mor R, Kushnir I, Meyer JJ, Ekstein J, Ben-Dov I. Breath sound distribution images of patients with pneumonia and pleural effusion. Respir Care. 2007;52: 1753-60.

21. Kramer MR, Raviv Y, Hardoff R, Shteinmatz A, Amital A, Shitrit D. Regional sound distribution analysis in single lung transplant recipients. J Heart Lung Transplant. 2007;26:1149-54.

22. Wang Z, Bartter T, Baumann B, Abouzgheib W, Chansky ME, Jean S. Asynchrony between left and right lung in acute asthma. J Asthma. 2008;45:575-8.

23. Maher TM, Gat M, Allen D, Devaraj A, Wells AU, Geddes DM. Reproducibility of dynamically represented acoustic lung images from healthy individuals. Thorax. 2008;63:542-8.

24. Comce F, Bingol Z, Kiyan E, Tanju S, Toker A, Cagatay P, et al. Vibrationresponse imaging versus quantitative perfusion scintigraphy in the selection of patients for lung-resection surgery. Respir Care. 2011;56:1936-41.

25. Luiz RR, Szklo M. More than one statistical strategy to assess agreement of quantitative measurements may usefully be reported. J Clin Epidemiol. 2005; 58:215-6. 